UDC 539.17

\title{
Whittaker asymptotics of shell-model wave functions for excited states of ${ }^{13} \mathrm{C}$
}

\author{
N.A. Burkova ${ }^{1 *}$, N.V. Afanasyeva ${ }^{2}$, D.N. Sharafutdinova ${ }^{1}$ \\ ${ }^{1}$ Institute of Experimental and Theoretical Physics, Al-Farabi Kazakh National University, \\ 71, al-Farabi Ave., 050040, Almaty, Kazakhstan \\ ${ }^{2}$ Institute of Nuclear Physics, 1, Ibragimov Str., 050032, Almaty, Kazakhstan \\ *e-mail: natali.burkova@gmail.com
}

\begin{abstract}
Halo and skin structure of $1 / 2^{+}, 3 / 2^{+}, 5 / 2^{+}$excited states of ${ }^{13} \mathrm{C}$ nucleus is under investigation. The modified radial functions have been constructed basing on the shell model functions. The matching procedure was performed while using the corresponding Whittaker exponential-like asymptotics. To find the matching radius via the oscillator parameter $r_{0}$ three options were examined related to the experimental and theoretical data on the mean square radii, and uncertainty relation. The renormalization of the modified functions shows the input of the asymptotic region near $15-20 \%$. The most qualitative effect is the redistribution of the probability density both at short and long-range distances. The obtained functions are given as parametrization by Gauss basis. It is proposed to confirm the observed halo and skin states of ${ }^{13} \mathrm{C}$ via the impulse distributions measured with different probing projectile like high-energy protons or $\alpha$-particles. The most probable halo effect the $2 \mathrm{~S}$-state reveals due to the node behavior of the corresponding radial wave function.
\end{abstract}

Key words: halo nuclei, skin state, shell model, oscillator parameter, Whittaker function, matching radius.

PACS: 24.10

\section{Introduction}

Currently, vast experimental material is assembled on the study the unusual properties of some atomic nuclei, known as the halo-states [1-5]. The defining feature of a halo was from the beginning understood to be a large spatial extension caused by neutrons tunneling out from a nuclear core. Nuclei may reveal not only well-developed halo states but also something intermediate between normal nuclei and halo nuclei, so called skin-states.

Halo effect research attractiveness that is quite enough reliably identify two main features of halo states as large root mean radius $r_{m s}$ and narrow localized impulse distributions. These two characteristics, obviously, related to each other according the Heisenberg uncertainty relation $(\Delta x \Delta p \sim \hbar)$.

Despite the seeming simplicity and transparency of the question, both experimental and theoretical studies still face certain difficulties. First of all, it is ambiguity on rms radii data [6-7]. The difficulty lies in the fact that the lifetime of most neutron-rich isotopes is extremely short and is typically a few milliseconds. It is therefore to work with short-lived isotopes needed entirely new technique, compared with conventional ones.
Recent advances are related to the collinear laser beams and anti-collinear technologies (CACLB) of the European Organization for Nuclear Research. Today, the installation ISOLDE at CERN synthesized radioactive isotopes up to $Z=10$ in a collision energy of $1.4 \mathrm{GeV}$ protons on uranium carbide target. Currently, it reports on the results of measurements of the charge and mass and radii for ${ }^{7,9,10} \mathrm{Be}$ isotopes by laser spectroscopy [8]. With great precision, it was found that ${ }^{11} \mathrm{Be}$ is a halo-nucleus with a radius of $7 \mathrm{fm}$, to compare ${ }^{10} \mathrm{Be}$ isotope core radius is $2.5 \mathrm{fm}$. Experiments are planned to heavier isotopes, particularly for the carbon-oxygen group.

Theoretical models deal with the description of the nuclei with prominent $\alpha$ - cluster structure, which include the majority of $p$-shell nuclei, may be represented as a semi-phenomenological and microscopic. The first, are the cluster models and their various modifications [9]. To the second ones may refer the models such as the resonating group method (RGM), the method of K-harmonics, variational Monte Carlo (VMC), the method of fermion molecular dynamics (FMD), and others. It is believed that microscopic models not contain adjustable parameters. However, they are based on the nucleon-nucleon potentials, which, in turn, are fitted according to the elastic $N N$ - scattering in a 
wide range of energies, so there are parameters a lot.

At the same time almost all the model approaches are based exactly on the data on the shell structure of atomic nuclei, as on the comparative, which are considered at the present time as the classic ones and obtained in the framework of the shell model (SM), which has only one oscillator parameter $r_{0}$.

For the halo-states the radial functions that characterize the distribution of the probability density, can be divided into internal and external conventional parts. The first is a model-depending, and the second asymptotic one is model-independent. The last one determines the mean square $r_{m s}$ size of the nuclear system, as well as so-called asymptotic normalization coefficient (ANC), which can be measured experimentally [10-11]. All models somehow have to "converge" in the section point, call it a matching radius $r_{m}$ of these two areas. Thus, this is the main task to identify and substantiate the value of the $r_{m}$ radius.

Present paper reports on the modification of oscillatory wave functions for low-lying 2s-, 2dexcited states with $J^{\pi}=1 / 2^{+}, 3 / 2^{+}, 5 / 2^{+}$of ${ }^{13} \mathrm{C}$ nucleus. To construct the modified functions we have used the matching procedure of oscillatory wave functions with corresponding Whittaker functions.

In this context, the lower bound of the asymptotic region $r_{m}$ is defined, and it allows to answer a question about a pronounced halo, skin- or normal structure of these states. The advantage of using the shell model is that almost all the calculations can be carried out analytically.

\section{Modification procedure for the oscillator radial wave functions}

We are basing on the fundamental postulate of nonrelativistic quantum mechanics telling that functions to be regarded as wave functions (WF) should be square integrated, continuous, unambiguous, and final.

Assume the potential in Schrodinger radial equation is defined in two space intervals $0<r \leq r_{m}$ and $r_{m} \leq r<\infty$. Such a division is conventional, and depending on the properties of the treating microsystem may consist of more than two intervals. Let us restricted by one transition point.

The matching radius $r_{m}$ we can find unambiguously from the continuity condition

$$
\begin{cases}u_{1}\left(r_{m}\right)=u_{2}\left(r_{m}\right) & (a) \\ u_{1}^{\prime}\left(r_{m}\right)=u_{2}^{\prime}\left(r_{m}\right) & (b)\end{cases}
$$

Here $u_{1}(r)$ is any model function that should be modified providing the correct extended asymptotic behavior by some appropriate analytical $u_{2}(r)$ function. As $u_{1}(r)$ the shell model radial oscillator WF have been used $u_{1}(r)=R_{n \ell}(r)$ for the state with main quantum number $n$ and orbital momentum $\ell$. For $u_{2}(r)$ various functions with exponential asymptotic at $r \rightarrow \infty$ may be used. Here we are taking $W_{0, \ell}(z)$ Whittaker functions [12] as $u_{2}(r)$

$$
u_{2}(r)=C_{n \ell} \frac{\sqrt{2 k_{0}}}{r} W_{0, \ell}(z),
$$

where $z=2 k_{0} r-$ dimensionless variable, $k_{0}$ - wave number. Factor $C_{n \ell}$ is asymptotic normalization coefficient.

To operate with functions of one and the same dimension expression (2) should be rewritten as the following

$$
\begin{gathered}
u_{2}(r)=C_{n \ell} \omega_{0, \ell}(r), \\
\omega_{0, \ell}(r)=\frac{\sqrt{2 k_{0}}}{r} W_{0, \ell}(z) .
\end{gathered}
$$

Therefore, the continuity condition (1) may be represented as equation of type

$$
\frac{R_{n \ell}^{\prime}(r)}{R_{n \ell}(r)}=\frac{\omega_{0, \ell}^{\prime}(r)}{\omega_{0, \ell}(r)},
$$

and the corresponding real roots define the matching radius $r_{m}$. The solutions may be found analytically or numerically. To find asymptotic constant $C_{n \ell}$ equation (1a) is used

$$
C_{n \ell}=\frac{R_{n \ell}\left(r_{m}\right)}{\omega_{0, \ell}\left(r_{m}\right)} .
$$

Basing on the obtained values of $r_{m}$ and $C_{n \ell}$, the modified radial channel functions are now defined as following

$$
R_{n \ell}^{m o d}(r)= \begin{cases}R_{n \ell}(r), & 0<r \leq r_{m} \\ C_{n \ell} \omega_{0, \ell}(r), & r_{m} \leq r<\infty\end{cases}
$$


The modified functions $R_{n \ell}^{\text {mod }}(r)$ should be normalized on to unit according the standard procedure

$$
N^{2} \int_{0}^{\infty}\left(R_{n \ell}^{\bmod }\right)^{2} r^{2} d r=1
$$

This expression has the explicit form while taking definition (6) into account:

$$
N^{2}\left[\int_{0}^{r_{m}} R_{n \ell}^{2} r^{2} d r+C_{n \ell}^{2} \int_{r_{m}}^{\infty} \omega_{0, \ell}^{2} r^{2} d r\right]=1 .
$$

To continue our notations let us introduce $I_{1}$ and $I_{2}$ for the first and second integrals in (8) respectively, then the normalizing constant $N$ is

$$
N=\frac{1}{\sqrt{I_{1}+C_{n \ell}^{2} I_{2}}}
$$

Finally the normalized modified radial function is defined as $\tilde{R}_{n \ell}^{\text {mod }}(r)=N \cdot R_{n \ell}^{\text {mod }}(r)$, or taking into account (6) one arrives

$$
\tilde{R}_{n \ell}^{\text {mod }}(r)= \begin{cases}N \cdot R_{n \ell}(r), & 0<r \leq r_{m} \\ N \cdot C_{n \ell} \omega_{0, \ell}(r), & r_{m} \leq r<\infty\end{cases}
$$

It is formal to somewhat extent, but it makes obvious that all relations for obtaining of matching radius $r_{m}$ and ANC $C_{n \ell}$ preserve.

To characterize the probability density in interior and exterior regions it is natural to introduce the corresponding weights

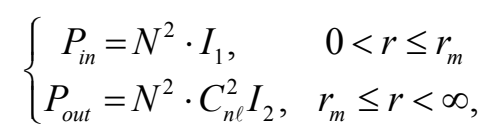

there relative probabilities $P_{\text {in }}$ and $P_{\text {out }}$ will be given in $\%$.

For completeness let us present the analytical expression for the Whittaker functions (3) obtained for the special case of zero Coulomb potential

$$
W_{0, l}(z)=e^{-z / 2} \sum_{n=0}^{l} z^{-n} \frac{(l+n) !}{(l-n) ! n !} .
$$

\section{Modified functions in space representation}

Here we are giving the results of the calculations for the modified radial WF of the low-lying excited states $J^{\pi}=1 / 2^{+}, 3 / 2^{+}$and $5 / 2^{+}$of positive parity $\pi$ with total momentum $J$ in ${ }^{13} \mathrm{C}$ nucleus corresponding to the $s^{4} p^{8}(2 \mathrm{~s}-1 \mathrm{~d})^{1}$ configuration with ${ }^{12} \mathrm{C}$ as a core and a valence neutron in $2 \mathrm{~s}-1 \mathrm{~d}$ shell. The choice of these states is due to the fact that they are the real candidates for the manifestation of skin or halo structure on grounds such as large rms radii $r_{m s}$, small binding energy of the valence neutron $\varepsilon=\hbar^{2} k_{0}^{2} / 2 \mu$.

For the first excited state $J^{\pi}=1 / 2^{+}$with excitation energy $\quad E_{\text {exc }}=3,089 \quad \mathrm{MeV} \quad$ oscillator $R_{2 s}(r)=\frac{\sqrt{6}}{\pi^{1 / 4}} \cdot \frac{1}{r_{0}^{3 / 2}}\left(1-\frac{2 r^{2}}{3 r_{0}^{2}}\right) e^{-\frac{r^{2}}{2 r_{0}^{2}}}$ function was used. For the other $J^{\pi}=5 / 2^{+}$and $3 / 2^{+}$states with the excitation energies $E_{e x c}=3,854$ and $7,67 \mathrm{MeV}$ $R_{2 d}(r)=\frac{4}{\pi^{1 / 4} \sqrt{15}} \cdot \frac{r^{2}}{r_{0}^{7 / 2}} e^{-\frac{r^{2}}{2 r_{0}^{2}}}$ radial function was employed.

Let us define how the appropriate parameters necessary for the modification procedure may be determined.

Method №1 consider the parameter $k_{0}$ to be known from the relation $\varepsilon=\hbar^{2} k_{0}^{2} / 2 \mu$. Then using $\rho_{0}$ as the solution of equation (4) and relation $\rho_{0}=k_{0} r_{m}$, the matching radius $r_{m}$ may be found. Finally the oscillator parameter we can obtain from $r_{0}=1 / k_{0}$.

Method №2 for the definition of oscillator parameter is basing on the known correlation for the 3D harmonic oscillator $\overline{r^{2}}=r_{0}^{2} \cdot(n+3 / 2)$, using the rms value $r_{m s}$ the oscillator parameter $r_{0}$ may be obtained. The matching radius $r_{m}$ is coming from the relation $\rho_{0}=r_{m} / r_{0}$.

We obtained the numerical values: $\rho_{0}=2,474556$ for $R_{2 s}$ matching function as well as $\rho_{0}=2,513260$ in case of $R_{2 d}$. Table 1 is a summary of all obtained parameters with pointed above method in last column.

The resulting modified functions in space representation have been expanded by Gaussian basis. In case of $J^{\pi}=1 / 2^{+}$state the expansion coefficients are given in table 2 for the corresponding function of the form

$$
\tilde{R}_{2 s}^{\text {mod }}(r)=\sum_{i=1}^{15} B_{i} \cdot e^{-\beta_{i} \vec{r}^{2}} .
$$

In case of $J^{\pi}=5 / 2^{+}$and $3 / 2^{+}$states the corresponding expanded function is of the form

$$
\tilde{R}_{2 d}^{\text {mod }}(r)=r^{2} \sum_{i=1}^{15} D_{i} \cdot e^{-\gamma_{i} \vec{r}^{2}}
$$

Corresponding expansion coefficients are given in tables 3 and 4. 
Table 1 - Parameters of excited states $J^{\pi}=1 / 2^{+}, 5 / 2^{+}, 3 / 2^{+}$of ${ }^{13} \mathrm{C}$

\begin{tabular}{|c|c|c|c|c|c|}
\hline$J^{\pi}$ & $E_{e x c}, \mathrm{MeV}$ & $r_{r m s}, f m$ & $r_{m}, f m$ & $r_{0}, f m$ & № \\
\hline \multirow{2}{*}{$1 / 2^{+}$} & \multirow{2}{*}{3,089} & \multirow{2}{*}{$5,04 \pm 0,75$} & 6,669 & 2,695 & 2 \\
\hline & & & 8,604 & 3,477 & 1 \\
\hline \multirow{2}{*}{$5 / 2^{+}$} & \multirow{2}{*}{3,874} & \multirow{2}{*}{$3,68 \pm 0,4$} & 4,946 & 1,968 & 2 \\
\hline & & & 11,393 & 4,533 & 1 \\
\hline $3 / 2^{+}$ & 7,67 & - & 7,218 & 2,872 & 1 \\
\hline
\end{tabular}

Table 2 - Expansion coefficients for $1 / 2^{+}$excited state of ${ }^{13} \mathrm{C}$ corresponding data of Table 1

\begin{tabular}{|c|c|c|c|c|}
\hline$i$ & № 2 $\beta_{i}$ & $B_{i}$ & №1 $\beta_{i}$ & $B_{i}$ \\
\hline 1 & $0.995675768 \mathrm{D}-02$ & $-0.214390442 \mathrm{D}-01$ & $0.779153573 \mathrm{D}-02$ & $-0.157705075 \mathrm{D}-01$ \\
\hline 2 & $0.336098351 \mathrm{D}-01$ & $-0.189661409 \mathrm{D}+00$ & $0.263009545 \mathrm{D}-01$ & $-0.320160296 \mathrm{D}+00$ \\
\hline 3 & $0.599294538 \mathrm{D}-01$ & $-0.190958104 \mathrm{D}+00$ & $0.468970417 \mathrm{D}-01$ & $0.188133220 \mathrm{D}+01$ \\
\hline 4 & $0.889974970 \mathrm{D}-01$ & $0.464299511 \mathrm{D}+01$ & $0.696438740 \mathrm{D}-01$ & $-0.142428669 \mathrm{D}+02$ \\
\hline 5 & $0.121524851 \mathrm{D}+00$ & $-0.323408024 \mathrm{D}+02$ & $0.950977468 \mathrm{D}-01$ & $0.577866466 \mathrm{D}+02$ \\
\hline 6 & $0.158690793 \mathrm{D}+00$ & $0.104349783 \mathrm{D}+03$ & $0.124181488 \mathrm{D}+00$ & $-0.133732675 \mathrm{D}+03$ \\
\hline 7 & $0.202296685 \mathrm{D}+00$ & $-0.185342008 \mathrm{D}+03$ & $0.158304731 \mathrm{D}+00$ & $0.201189391 \mathrm{D}+03$ \\
\hline 8 & $0.255142402 \mathrm{D}+00$ & $0.204894845 \mathrm{D}+03$ & $0.199658483 \mathrm{D}+00$ & $-0.205576264 \mathrm{D}+03$ \\
\hline 9 & $0.321792941 \mathrm{D}+00$ & $-0.147094874 \mathrm{D}+03$ & $0.251815026 \mathrm{D}+00$ & $0.144536068 \mathrm{D}+03$ \\
\hline 10 & $0.410216901 \mathrm{D}+00$ & $0.688772842 \mathrm{D}+02$ & $0.321010086 \mathrm{D}+00$ & $-0.694744674 \mathrm{D}+02$ \\
\hline 11 & $0.535673529 \mathrm{D}+00$ & $-0.205394784 \mathrm{D}+02$ & $0.419184595 \mathrm{D}+00$ & $0.222793718 \mathrm{D}+02$ \\
\hline 12 & $0.731454805 \mathrm{D}+00$ & $0.368965561 \mathrm{D}+01$ & $0.572390775 \mathrm{D}+00$ & $-0.454905888 \mathrm{D}+01$ \\
\hline 13 & $0.108623797 \mathrm{D}+01$ & $-0.359689964 \mathrm{D}+00$ & $0.850021890 \mathrm{D}+00$ & $0.545615092 \mathrm{D}+00$ \\
\hline 14 & $0.193686319 \mathrm{D}+01$ & $0.155681470 \mathrm{D}-01$ & $0.151566798 \mathrm{D}+01$ & $-0.333966034 \mathrm{D}-01$ \\
\hline 15 & $0.653803936 \mathrm{D}+01$ & $-0.195974076 \mathrm{D}-03$ & $0.511626062 \mathrm{D}+01$ & $0.835301964 \mathrm{D}-03$ \\
\hline
\end{tabular}

Table 3 - Expansion coefficients for $5 / 2^{+}$excited state of ${ }^{13} \mathrm{C}$ corresponding data of Table 1

\begin{tabular}{|c|c|c|c|c|}
\hline$i$ & \multicolumn{2}{|c|}{ №2 $\gamma_{i} D_{i}$} & \multicolumn{2}{c|}{ № $1 \gamma_{i} D_{i}$} \\
\hline 1 & $0.934423119 \mathrm{D}-02$ & $0.193301166 \mathrm{D}-04$ & $0.507316101 \mathrm{D}-02$ & $0.153278531 \mathrm{D}-04$ \\
\hline 2 & $0.282397165 \mathrm{D}-01$ & $0.102243489 \mathrm{D}-02$ & $0.171248624 \mathrm{D}-01$ & $0.107571441 \mathrm{D}-02$ \\
\hline 3 & $0.477749532 \mathrm{D}-01$ & $-0.839234710 \mathrm{D}-02$ & $0.305352182 \mathrm{D}-01$ & $-0.293365722 \mathrm{D}-02$ \\
\hline 4 & $0.684424019 \mathrm{D}-01$ & $0.899360796 \mathrm{D}-01$ & $0.453459496 \mathrm{D}-01$ & $0.570832256 \mathrm{D}-01$ \\
\hline 5 & $0.908476504 \mathrm{D}-01$ & $-0.425774826 \mathrm{D}+00$ & $0.619192670 \mathrm{D}-01$ & $-0.242640999 \mathrm{D}+00$ \\
\hline 6 & $0.115788434 \mathrm{D}+00$ & $0.118540758 \mathrm{D}+01$ & $0.808560348 \mathrm{D}-01$ & $0.616231656 \mathrm{D}+00$ \\
\hline 7 & $0.144383322 \mathrm{D}+00$ & $-0.156836386 \mathrm{D}+01$ & $0.103074082 \mathrm{D}+00$ & $-0.103888477 \mathrm{D}+01$ \\
\hline 8 & $0.178298554 \mathrm{D}+00$ & $-0.991321547 \mathrm{D}-01$ & $0.129999999 \mathrm{D}+00$ & $0.120998646 \mathrm{D}+01$ \\
\hline 9 & $0.220180378 \mathrm{D}+00$ & $0.411323404 \mathrm{D}+01$ & $0.163959740 \mathrm{D}+00$ & $-0.988919594 \mathrm{D}+00$ \\
\hline 10 & $0.274555699 \mathrm{D}+00$ & $-0.670385983 \mathrm{D}+01$ & $0.209013461 \mathrm{D}+00$ & $0.567618348 \mathrm{D}+00$ \\
\hline 11 & $0.349930621 \mathrm{D}+00$ & $0.537336858 \mathrm{D}+01$ & $0.272936045 \mathrm{D}+00$ & $-0.226320361 \mathrm{D}+00$ \\
\hline 12 & $0.464483627 \mathrm{D}+00$ & $-0.243539615 \mathrm{D}+01$ & $0.372690399 \mathrm{D}+00$ & $0.613817482 \mathrm{D}-01$ \\
\hline 13 & $0.665419299 \mathrm{D}+00$ & $0.632727569 \mathrm{D}+00$ & $0.553459300 \mathrm{D}+00$ & $-0.110395726 \mathrm{D}-01$ \\
\hline 14 & $0.112573289 \mathrm{D}+01$ & $-0.935347961 \mathrm{D}-01$ & $0.986869335 \mathrm{D}+00$ & $0.132846869 \mathrm{D}-02$ \\
\hline 15 & $0.340214046 \mathrm{D}+01$ & $0.112954444 \mathrm{D}-01$ & $0.333125777 \mathrm{D}+01$ & $-0.177390635 \mathrm{D}-03$ \\
\hline
\end{tabular}


Table 4 -Expansion coefficients for $3 / 2^{+}$excited state of ${ }^{13} \mathrm{C}$ corresponding data of Table 1

\begin{tabular}{|c|c|c|c|c|c|}
\hline$i$ & №1 $\gamma_{i}$ & $D_{i}$ & $i$ & $\gamma_{i}$ & $D_{i}$ \\
\hline 1 & $0.585738861 \mathrm{D}-02$ & $0.118469219 \mathrm{D}-05$ & 9 & $0.189305230 \mathrm{D}+00$ & $0.107028918 \mathrm{D}+01$ \\
\hline 2 & $0.197720856 \mathrm{D}-01$ & $0.309624471 \mathrm{D}-03$ & 10 & $0.241323519 \mathrm{D}+00$ & $-0.114006343 \mathrm{D}+01$ \\
\hline 3 & $0.352554628 \mathrm{D}-01$ & $-0.523338692 \mathrm{D}-03$ & 11 & $0.315127487 \mathrm{D}+00$ & $0.713878510 \mathrm{D}+00$ \\
\hline 4 & $0.523556906 \mathrm{D}-01$ & $0.195186077 \mathrm{D}-01$ & 12 & $0.430302230 \mathrm{D}+00$ & $-0.278365868 \mathrm{D}+00$ \\
\hline 5 & $0.714909714 \mathrm{D}-01$ & $-0.737727901 \mathrm{D}-01$ & 13 & $0.639015042 \mathrm{D}+00$ & $0.682627507 \mathrm{D}-01$ \\
\hline 6 & $0.933550533 \mathrm{D}-01$ & $0.212603276 \mathrm{D}+00$ & 14 & $0.113942317 \mathrm{D}+01$ & $-0.108208117 \mathrm{D}-01$ \\
\hline 7 & $0.119007646 \mathrm{D}+00$ & $-0.110494484 \mathrm{D}+00$ & 15 & $0.384621565 \mathrm{D}+01$ & $0.184247221 \mathrm{D}-02$ \\
\hline 8 & $0.150095869 \mathrm{D}+00$ & $-0.452967220 \mathrm{D}+00$ & & & \\
\hline
\end{tabular}

Table 5 - Characteristics of radial modified wave functions $\tilde{R}_{2 l}^{\text {mod }}(r)$

\begin{tabular}{|c|c|c|c|c|}
\hline \multirow{2}{*}{$J^{\pi}, \tilde{R}_{2 l}^{\text {mod }}(r)$} & $r_{m}, f m$ & $C_{2 \ell}$ & $N$ & $P_{\text {out }}, \%$ \\
\hline \multirow{2}{*}{$1 / 2^{+}, \tilde{R}_{2 s}^{\text {mod }}(r)$} & 6,669 & 0,278476 & 0,940277 & 24,9 \\
\cline { 2 - 5 } & 8,604 & 0,181369 & 0,967572 & 20,1 \\
\hline \multirow{2}{*}{$5 / 2^{+}, \tilde{R}_{2 d}^{\text {mod }}(r)$} & 4,946 & 3,759468 & 0,979647 & 15,7 \\
\hline \multirow{2}{*}{$3 / 2^{+}, \tilde{R}_{2 d}^{\text {mod }}(r)$} & 11,393 & 0,584071 & 0,958564 & 10,5 \\
\hline
\end{tabular}

Table 5 is a short summary on the general characteristics of the obtained modified radial functions $\tilde{R}_{2 l}^{\text {mod }}(r)$. Let us pay attention to the probability of the localization of the valence neutron out of the mean square area of the core ${ }^{12} \mathrm{C}$ given as $P_{\text {out }}(\%)$. Comparison of these values make it possible to conclude that $1 / 2^{+}$state is the halo one, but states $5 / 2^{+}$ and $3 / 2^{+}$are likely belong to the skin structure states.

\section{Modified functions in impulse representation}

Due to the Heisenberg uncertainty relation "coordinate-momentum" all modifications of the wave functions at large distances must, first of all reflected in the momentum distributions for small values $q$.

The corresponding analytical expressions for the oscillator and modified functions obtained on the base of (13) in impulse representation for the case $J^{\pi}=1 / 2^{+}$are:

$$
\begin{gathered}
R_{2 s}(q)=r_{0}^{3 / 2} \frac{\sqrt{6}}{\pi^{1 / 4}} \cdot\left(1-\frac{2 q^{2} r_{0}^{2}}{3}\right) e^{-\frac{q^{2} r_{0}^{2}}{2}} \\
R_{2 s}^{\bmod }(q)=\frac{\sqrt{\pi}}{4} \sum_{i=1}^{15} B_{i} \cdot \frac{1}{\beta_{i}^{3 / 2}} e^{-\frac{q^{2}}{4 \beta_{i}}}
\end{gathered}
$$

For excited states $5 / 2^{+}$and $3 / 2^{+}$the analogue expressions for the oscillator and modified functions on the base of (14) have been obtained

$$
\begin{gathered}
R_{2 d}(q)=r_{0}^{3 / 2} \frac{4}{\pi^{1 / 4} \sqrt{15}} \cdot q^{2} r_{0}^{2} e^{-\frac{q^{2} r_{0}^{2}}{2}} \\
R_{2 d}^{\bmod }(q)=\frac{\sqrt{\pi}}{16} \sum_{i=1}^{15} D_{i} \cdot \frac{q^{2}}{\gamma_{i}^{7 / 2}} e^{-\frac{q^{2}}{4 \gamma_{i}}} .
\end{gathered}
$$

It is natural to discuss the probability density constructed as squared functions (15)-(18) $\rho_{2 l}(q)=\left|R_{2 l}(q)\right|^{2}$. The results of calculations are given in Figures $1-3$. 

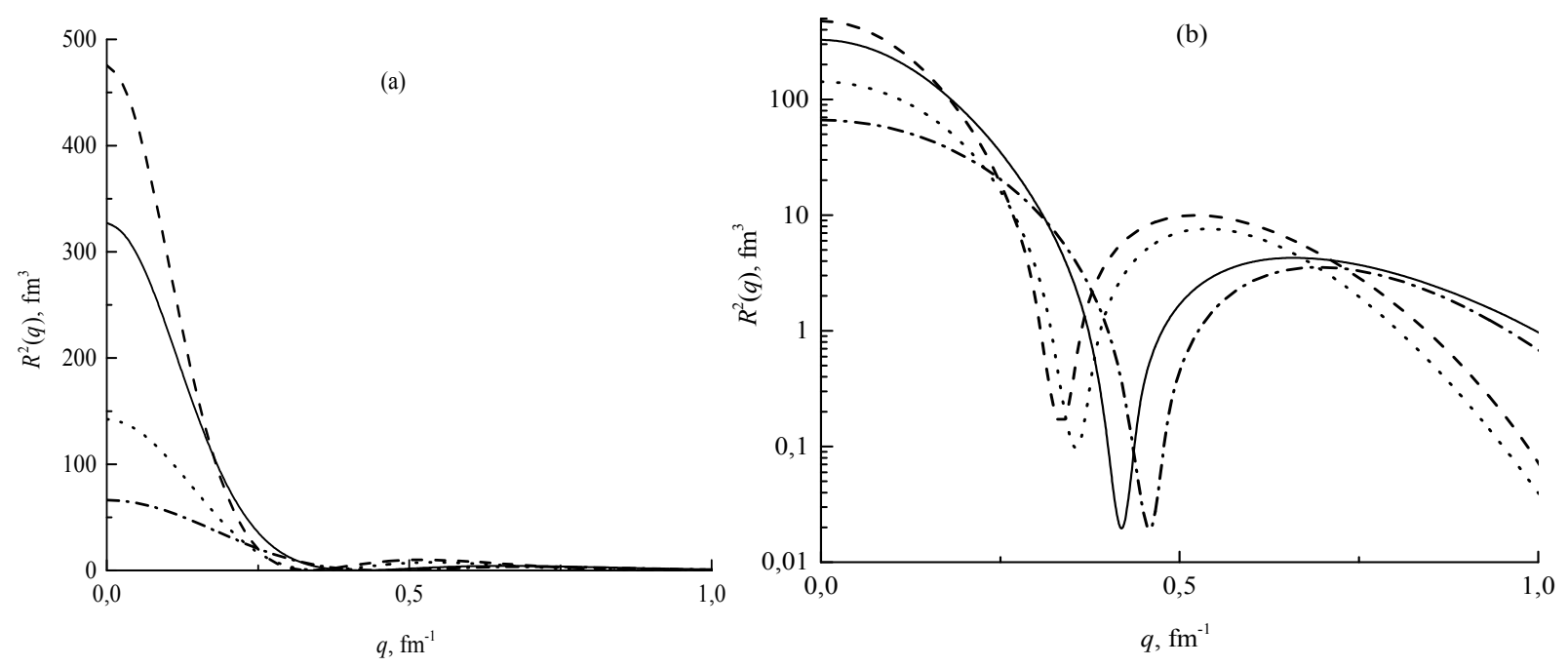

Figure 1 - Impulse distributions for excited $1 / 2^{+}$state of ${ }^{13} \mathrm{C}$ : (a) - linear scale; (b) - logarithmic scale. Set №2: $\rho_{2 s}^{\bmod }(q)$ - solid line, $\rho_{2 s}(q)$ - dash-dot; set №1: $\rho_{2 s}^{\text {mod }}(q)$ - long dash, $\rho_{2 s}(q)-$ dots

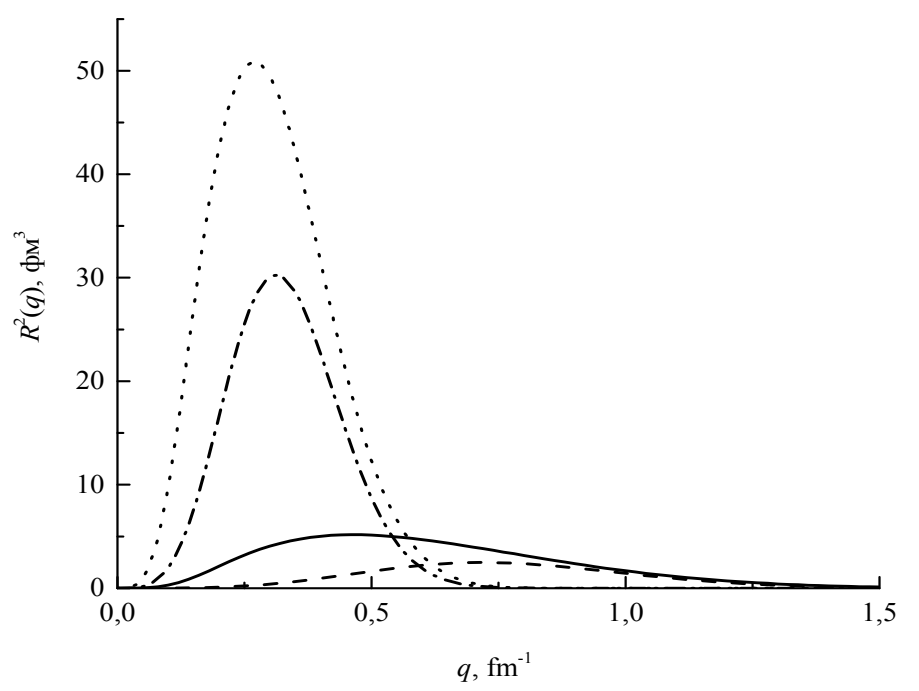

Figure 2 - Impulse distributions for excited $5 / 2^{+}$state of ${ }^{13} \mathrm{C}$. Set №2: $\rho_{2 d}^{\bmod }(q)-$ solid line, $\rho_{2 d}(q)$ - dash; set №1: $\rho_{2 d}(q)$ - dash-dot, $\rho_{2 d}^{\bmod }(q)-\operatorname{dots}$ 


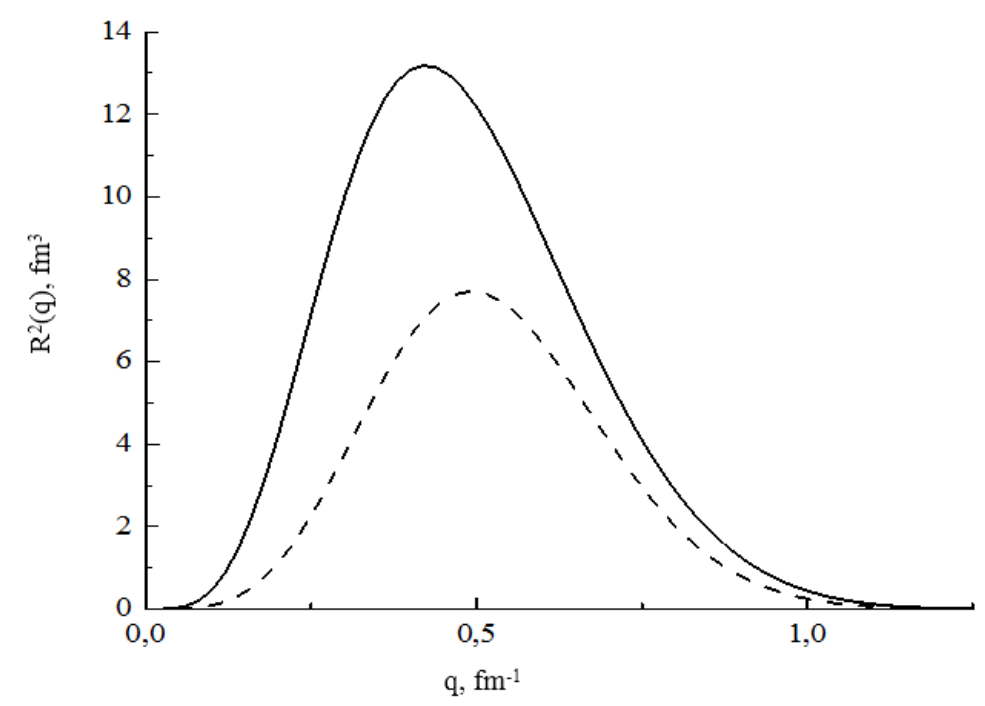

Figure 3 - Impulse distributions for excited $3 / 2^{+}$state of ${ }^{13} \mathrm{C}$.

$$
\rho_{2 d}^{\bmod }(q)-\text { solid line, } \rho_{2 d}(q)-\text { dash }
$$

As can be clearly seen from Figure 1, in fact, in the region of small momentum transfers $q \rightarrow 0$ momentum distributions obtained for all functions differ drastically. Thus, our assumption is that this characteristic is sensitive to the modifications of the asymptotic behavior is fully confirmed.

Figures 2 and 3 correspond the nodeless functions. They are exactly equal to zero at $q=0$. Such momentum distributions are compared by a well-known characteristic of the width at half-of height maximum $\Gamma$. It is for the $\Gamma$ values can be seen how wide or narrow impulse distributions, and, consequently, to draw conclusions about the asymptotic behavior of the length of the asymptotic in the coordinate representation.

\section{Conclusions}

Sufficiently general method of the modification of radial functions for the improving of the asymptotic behavior is presented. The coming ambiguities appearing while defining the oscillator parameters have been examined. The modified functions are presented as convenient expansion by Gaussian basis. The different versions of the modifications №1 and 2, as well as purely oscillator functions significantly distanced themselves in the momentum representation. To address the issue of choice of reliable functions it is necessary in the future to compare the obtained results with the available experimental data on elastic and inelastic form factors.

\section{References}

1 I. Tanihata, S. Terashima, R. Kanungo. Observation of large enhancements of charge exchange cross section with neutron-rich carbon isotopes // Phys. Rev. - 2016. - Vol. 4 - P. 1-11.

2 P.G. Hansen. Nuclear halos // Rev. Nucl. Part. Sci. - 1995 - Vol. 45. - P. 591-634.

3 I. Tanihataa, H. Savajols, R. Kanungod. Recent experimental progress in nuclear halo structure studies // Prog. Part. Nucl. Phys. - 2013. - Vol. 68. - P. 215-313

4 W. Horiuchi, Y. Suzuki, B. Abu-Ibrahim. Systematic analysis of reaction cross section of carbon isotopes // Phys. Rev. - 2007 - Vol. C75 - P. 044607 $-1-14$.
5 A.R. Samana, T. Tarutina, M.S. Hussein. Pairing correlations in odd-mass carbon isotopes and effect of Pauli principle in particle-core coupling in ${ }^{13} \mathrm{C}$ and ${ }^{11} \mathrm{Be}$ // Nuclear Physics - 2007 - Vol. A791. - P. 36-56.

6 T. L. Belyaeva, R. Perez-Torres, A. S. Demyanova et al. Neutron asymptotic normalization coefficients and halo radii of the first excited states of ${ }^{13} \mathrm{C}$ and ${ }^{11} \mathrm{Be} / /$ Phys. Rev. - 2014. - Vol. 90 - P. 1-2.

7 Lin Cheng-Jian et al. Extraction of the rms radius of ${ }^{13} \mathrm{C}$ from asymptotic normalization coefficients // Chinese Phys. Lett. - 2001. - Vol. 18. - P. 1446-1448. 
8 W. Nortershauser, D. Tiedemann, M. Zakov et al. Nuclear Charge Radii of ${ }^{7.9 .10} \mathrm{Be}$ and the one-neutron halo nucleus ${ }^{11} \mathrm{Be} / /$ Phys. Rev. Lett. - 2009. - Vol. 102. - P. 062503.

9 S.B. Dubovichenko. Thermonuclear Processes of the Universe. // First English edition. NOVA Sci. Publ. New York. - 2012. - P.194.

10 F. Cartoiu, L. Trache, C.A. Gagliardi et al. Radii of halo states in light nuclei deduced from ANC //
Romanian Reports in Physics. - 2007. - Vol. 59. No. 2. P. 357-375.

11 N. K. Timofeyuk, P. Descouvemont. Relation between widths of proton resonances and neutron asymptotic normalization coefficients in mirror states of light nuclei in a microscopic cluster model. // Phys. Rev. Lett. - 2013. - Vol. 91. - P. 2325019.

12 M. Abramowitz, I.A. Stegun. Handbook of Mathematical Functions. Washington: Nat. Bur. Stand. 1964. - P. 104 